\title{
SPDEF is required for mouse pulmonary goblet cell differentiation and regulates a network of genes associated with mucus production
}

\author{
Gang Chen,1 Thomas R. Korfhagen, ${ }^{1}$ Yan Xu, ${ }^{1}$ Joseph Kitzmiller, ${ }^{1}$ Susan E. Wert, ${ }^{1}$ Yutaka Maeda, ${ }^{1}$ \\ Alexander Gregorieff, ${ }^{2}$ Hans Clevers, ${ }^{2}$ and Jeffrey A. Whitsett ${ }^{1}$
}

\begin{abstract}
${ }^{1}$ The Perinatal Institute, Division of Pulmonary Biology, Cincinnati Children's Hospital Medical Center and the University of Cincinnati School of Medicine, Cincinnati, Ohio, USA. ${ }^{2}$ Netherlands Institute of Developmental Biology, Utrecht, The Netherlands.
\end{abstract}

\begin{abstract}
Various acute and chronic inflammatory stimuli increase the number and activity of pulmonary mucus-producing goblet cells, and goblet cell hyperplasia and excess mucus production are central to the pathogenesis of chronic pulmonary diseases. However, little is known about the transcriptional programs that regulate goblet cell differentiation. Here, we show that SAM-pointed domain-containing Ets-like factor (SPDEF) controls a transcriptional program critical for pulmonary goblet cell differentiation in mice. Initial cell-lineage-tracing analysis identified nonciliated secretory epithelial cells, known as Clara cells, as the progenitors of goblet cells induced by pulmonary allergen exposure in vivo. Furthermore, in vivo expression of SPDEF in Clara cells caused rapid and reversible goblet cell differentiation in the absence of cell proliferation. This was associated with enhanced expression of genes regulating goblet cell differentiation and protein glycosylation, including forkhead box A3 (Foxa3), anterior gradient 2 ( $\mathrm{Agr} 2$ ), and glucosaminyl ( $\mathrm{N}$-acetyl) transferase 3, mucin type (Gcnt3). Consistent with these findings, levels of SPDEF and FOXA3 were increased in mouse goblet cells after sensitization with pulmonary allergen, and the proteins were colocalized in goblet cells lining the airways of patients with chronic lung diseases. Deletion of the mouse Spdef gene resulted in the absence of goblet cells in tracheal/laryngeal submucosal glands and in the conducting airway epithelium after pulmonary allergen exposure in vivo. These data show that SPDEF plays a critical role in regulating a transcriptional network mediating the goblet cell differentiation and mucus hyperproduction associated with chronic pulmonary disorders.
\end{abstract}

\section{Introduction}

Goblet cell "hyperplasia" and mucus hypersecretion contribute to the pathogenesis of common pulmonary disorders, including asthma, chronic obstructive pulmonary disease, and cystic fibrosis (CF). Allergens, cigarette smoke, inhaled toxicants, and chronic infections induce mucus hyperproduction in conducting airways, causing airway obstruction and tissue remodeling. While initially derived from shared endodermal progenitors during lung morphogenesis (1), the conducting regions of the respiratory tract are lined by a diversity of epithelial cell types, including Clara, serous, basal, goblet (mucous), neuroepithelial, and ciliated cells, that together mediate innate host defense and mucociliary clearance to maintain sterility of the lung. While goblet cells are generally not abundant in the normal lung, goblet cell hyperplasia is induced by acute and chronic inflammation, influencing mucociliary clearance and innate host defense in the lung $(2,3)$. The differentiation of various pulmonary epithelial cell types is determined by both genetic and environmental factors that, in turn, regulate transcriptional programs controlling epithelial cell differentiation and behavior. During development, alveolar type II and Clara cell differentiation are dependent upon interactions of a number of transcription factors that regulate a group of genes mediating host defense and other aspects of lung function (4). In contrast, there is a paucity of knowledge regarding transcrip-

Conflict of interest: The authors have declared that no conflict of interest exists. Citation for this article: J. Clin. Invest. 119:2914-2924 (2009). doi:10.1172/JCI39731. tional programs regulating goblet cell differentiation. Goblet cells are found in many epithelial-enriched tissues where they synthesize, store, and secrete large mucopolysaccharide-rich mucins that influence mucociliary clearance and innate defense of the lung $(5,6)$. In the gastrointestinal tract, goblet cells are relatively abundant, and their differentiation is regulated by the Notch signaling pathway $(7,8)$. In the lung, goblet cells (mucous cells) are present in submucosal glands but are not abundant in conducting airways in the absence of inflammation. Numbers and activity of goblet cells are induced by a variety of acute and chronic inflammatory stimuli. Goblet cells are observed following pulmonary allergen sensitization, mediated primarily by the $\mathrm{TH}_{2}$-associated cytokines IL-4 and IL-13, which activate the IL-4 receptor, STAT6 phosphorylation, and subsequent gene expression (9-12). At the transcriptional level, pulmonary goblet cells are increased by allergens, dust mite, or IL-13 exposure and are associated with the decrease of forkhead box a2 (FOXA2) mRNA in bronchial and bronchiolar epithelial cells (13). In the mouse lung, deletion of the Foxa2 gene in respiratory epithelial cells is sufficient to induce goblet cell differentiation in vivo (14). The potential role of SAM-pointed domain-containing Ets-like factor (SPDEF), a member of the Ets family of transcription factors (15), in goblet cell differentiation is supported by the finding that SPDEF is induced following pulmonary allergen and IL-13 exposure (16). Chronic expression of SPDEF in epithelial cells of the mouse lung is associated with extensive goblet cell differentiation in the airways of transgenic mice (16). 
A

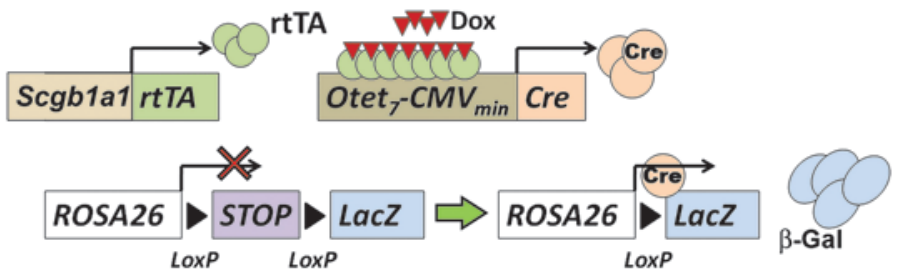

B
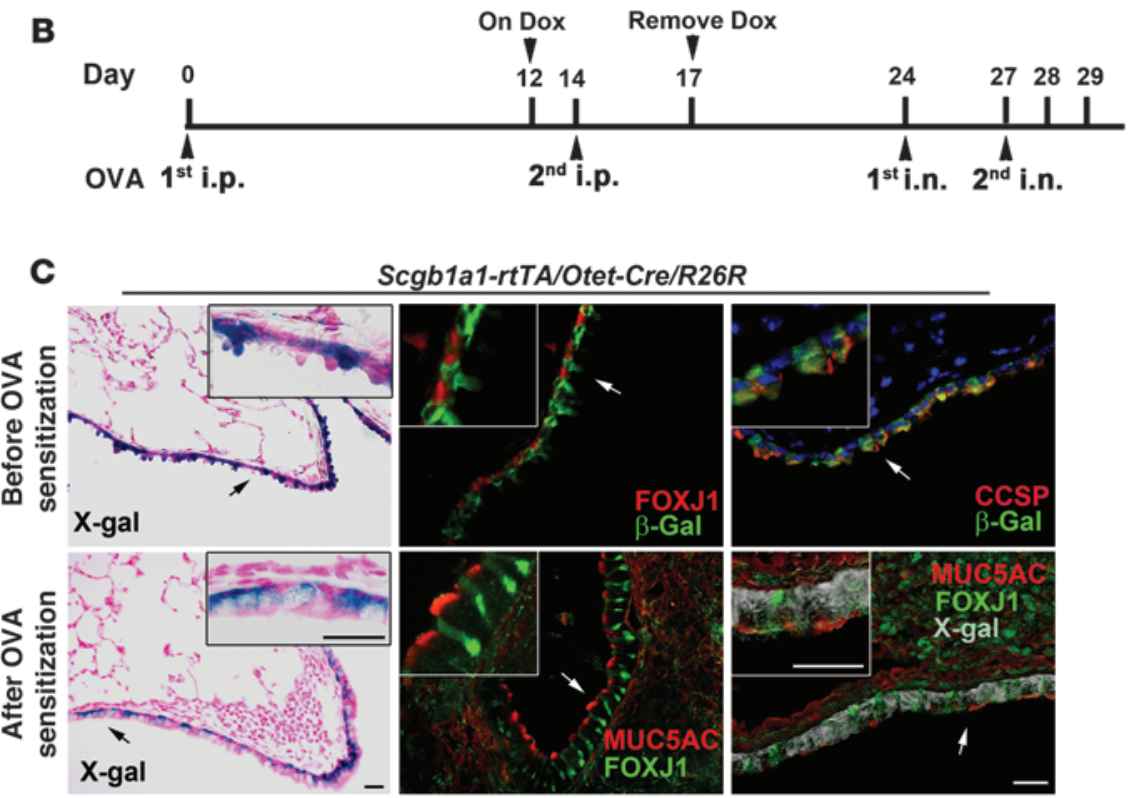

D

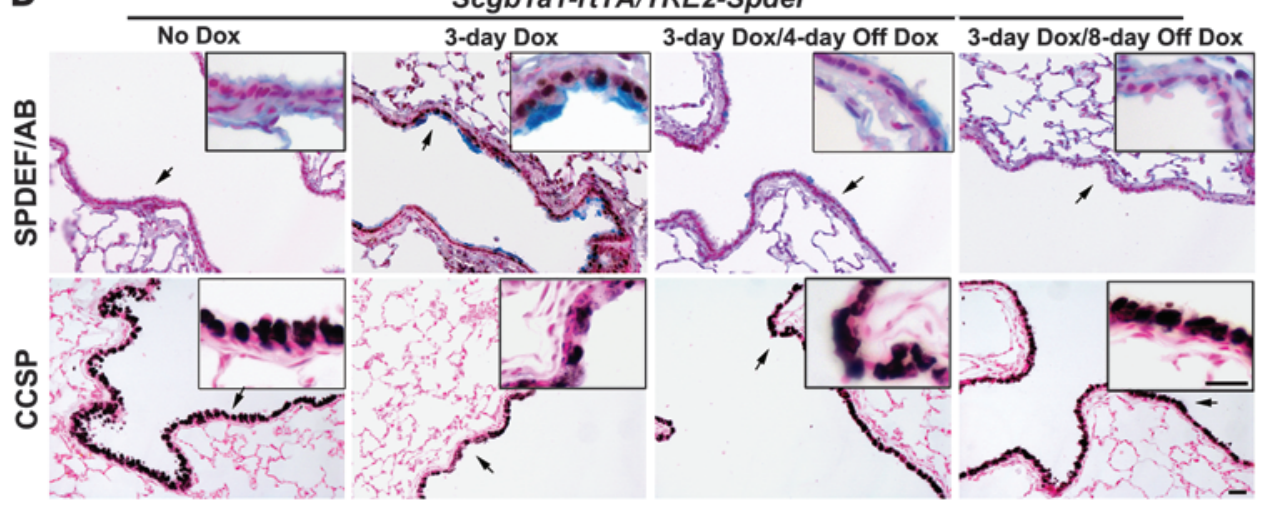

Figure 1

SPDEF caused differentiation of goblet cells from Clara cells. (A) Adult Scgb1a1-rtTA/Otet-Cre mice were mated to R26R mice. (B) To permanently label Clara cells, doxycycline (Dox) was administered from days 12 to 17 during i.p. sensitization with OVA. Mice were sacrificed either before receiving the first i.n. sensitization (day 24) to assess Clara cell labeling with $\beta$-gal or after the second i.n. sensitization (day 29) with OVA to induce goblet cell hyperplasia. (C) Before pulmonary OVA sensitization, $\beta$-gal was expressed in nonciliated epithelial cells, as shown by its exclusion from FOXJ1-expressing cells and colocalization with CCSP. After sensitization, $\beta$-gal was detected (white pseudocolor) in most goblet cells, identified by MUC5AC, indicating their derivation from Clara cells. MUC5AC, $\beta$-gal, and FOXJ1 were not colocalized. (D) Scgb1a1-rtTA/ TRE2-Spdef mice were treated 3 days with or without doxycycline. SPDEF induced goblet cell differentiation as detected by Alcian blue $(A B)$ and by changes in cell morphology. SPDEF staining decreased 4 and 8 days after withdrawal of doxycycline, at which time goblet cell differentiation was substantially resolved. CCSP was decreased in the conducting airway epithelium 3 days after induction of SPDEF. 4 to 8 days after withdrawal of doxycycline, CCSP staining was restored. Inserts show higher magnifications of the regions indicated by arrows. Scale bars: $25 \mu \mathrm{m}$.
The present study was undertaken to identify the role and mechanisms by which SPDEF regulates goblet cell differentiation in conducting airways of the lung. SPDEF was required for normal goblet cell differentiation in laryngeal-tracheal submucosal glands and in the bronchial-bronchiolar epithelium during experimental pulmonary allergen sensitization. SPDEF regulates a transcriptional network inhibiting Clara cell differentiation and inducing goblet cell differentiation and mucus production.

\section{Results}

Cellular origins of goblet cells induced by allergen exposure. To define the cellular precursors of goblet cells in the respiratory epithelium, lineage tracing was performed by conditionally labeling Clara cells using Cre recombinase to recombine the ROSA26 loxP/stop locus, thereby permanently expressing $\beta$-gal in Clara cells or their derivatives (Figure 1A). Administration of doxycycline to Scgb1a1-rtTA/ Otet-Cre/R26R (ROSA26 Cre reporter) transgenic mice caused extensive recombination in Clara cells within 5 days, at which time $\beta$-gal staining was confined to Clara cells and not detected in ciliated cells. Goblet cell differentiation was then induced by pulmonary sensitization with OVA (Figure 1B). The goblet cells induced by the allergen expressed $\beta$-gal, indicating their derivation from Clara cell progenitors (Figure 1C). Goblet cell differentiation, including increased MUC5AC staining (Figure 1C), occurred without evidence of cellular proliferation, as assessed by BrdU and phosphohistone H3 labeling (Supplemental Figure 1; supplemental material available online with this article; doi:10.1172/JCI39731DS1). Consistent with these findings, conditional expression of SPDEF 
A

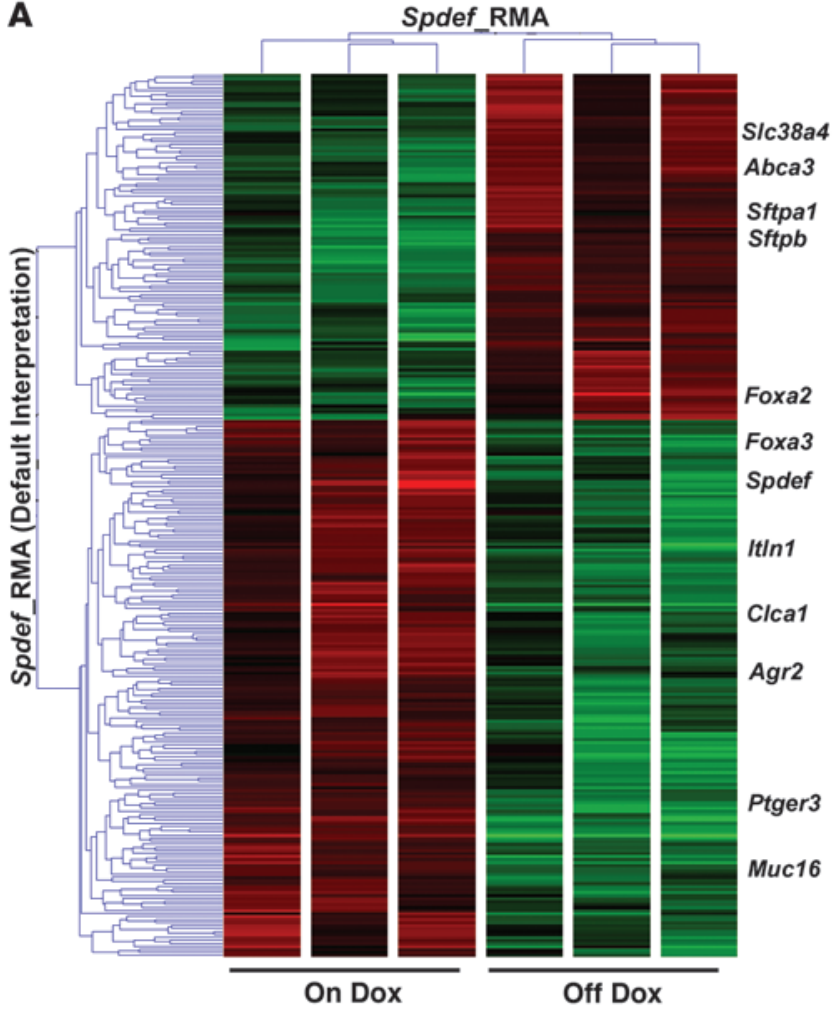

B

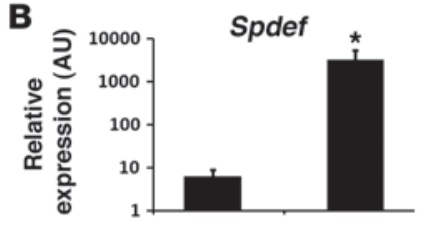

C

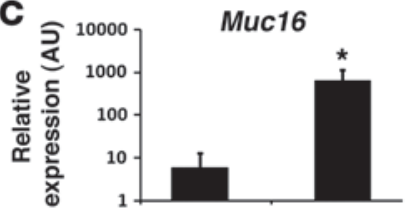

D

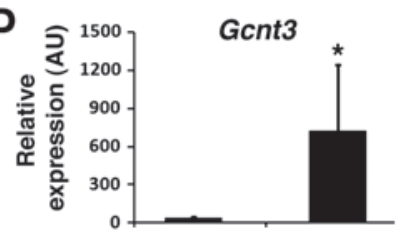

$\mathbf{E}$

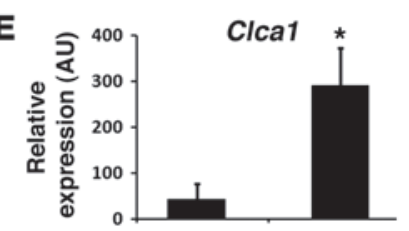

$\mathbf{F}$

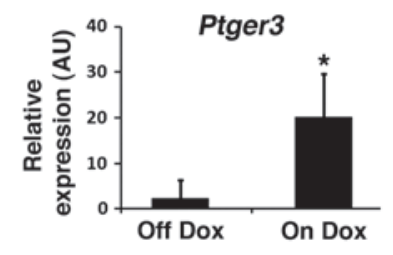

Figure 2

mRNA microarray analysis of bronchiolar epithelial cells: heat map and partial list of SPDEF-regulated genes. Bronchiolar cells were isolated by LCM and mRNAs isolated and subjected to mRNA microarray analysis after treating Scgb1a1-rtTA/TRE2-Spdef mice for 3 days with or without doxycycline. (A) Heat map of the mRNAs. Red indicates mRNAs increased by SPDEF; green indicates those decreased. A number of mRNAs that were previously associated with pulmonary allergen exposure, including Foxa3, Gcnt3, Clca1, Agr2, Ptger3, and Muc16 were induced by SPDEF. SPDEF inhibited genes selectively expressed in normal airway epithelial cells, including Abca3, Sttpa1, Sttpb, Sftpd, and Foxa2 (A). Quantitative RT-PCR was performed in triplicate using cDNAs obtained from bronchiolar cells by LCM. Spdef mRNA was induced by doxycycline treatment (B). SPDEF induced Muc16 (C), Gcnt3 (D), Clca1 (E), and Ptger3 (F) mRNAs. Results were expressed as mean \pm SD of 3 independent mice for each treatment. ${ }^{*} P<0.05$ versus offdoxycycline control littermates (Hsu's MCB test).

using the Clara cell-specific promoter (Scgb1a1-rtTA/TRE2-Spdef) induced marked goblet cell differentiation without causing proliferation in the conducting airways within 3 days. Neither BrdU labeling, phosphohistone $\mathrm{H} 3$, nor the number of cells per unit length of the bronchioles (latter data not shown) were increased during allergen sensitization or after expression of SPDEF in vivo. This process was rapidly reversible and associated with the restoration of Clara cell morphology and Clara cell secretory protein (CCSP) expression (Figure 1D). Thus, goblet cell differentiation in this model occurs without proliferation, a finding that does not support the term "hyperplasia."

SPDEF regulates gene expression in the respiratory epithelium. To identify mRNAs regulated by SPDEF during goblet cell differentiation, laser capture microdissection (LCM) was used to obtain and thyroid transcription factor 1 (TTF-1, also termed Nkx2-1), including ATP-binding cassette, subfamily A (ABC1), member 3 (Abca3), surfactant-associated protein A (Sftpa), Sftpb, Sftpd, P450 (cytochrome) oxidoreductase (Por), aquaporin 5 (Aqp5), and secretoglobin, family 1A, member 1 (uteroglobin) (Scgb1a1) $(26,27)$ (Figure 2A). SPDEF inhibited Scnn1b and Scnn 1g, consistent with a role for SPDEF in the regulation of fluid and electrolyte transport, which is important for mucociliary clearance in the lung. Quantitative RTPCR was used to confirm the SPDEF-related changes in a number of these mRNAs (Figure 2, B-F). Of note, mouse Clca1 mRNA was significantly induced by SPDEF in vivo. Human CLCA1, a calciumactivated chloride channel associated with mucus production (28), is the closest ortholog of mouse CLCA1 (29) (Swiss-Prot accession number Q9D7Z6). The finding that SPDEF inhibited Titf1 and 


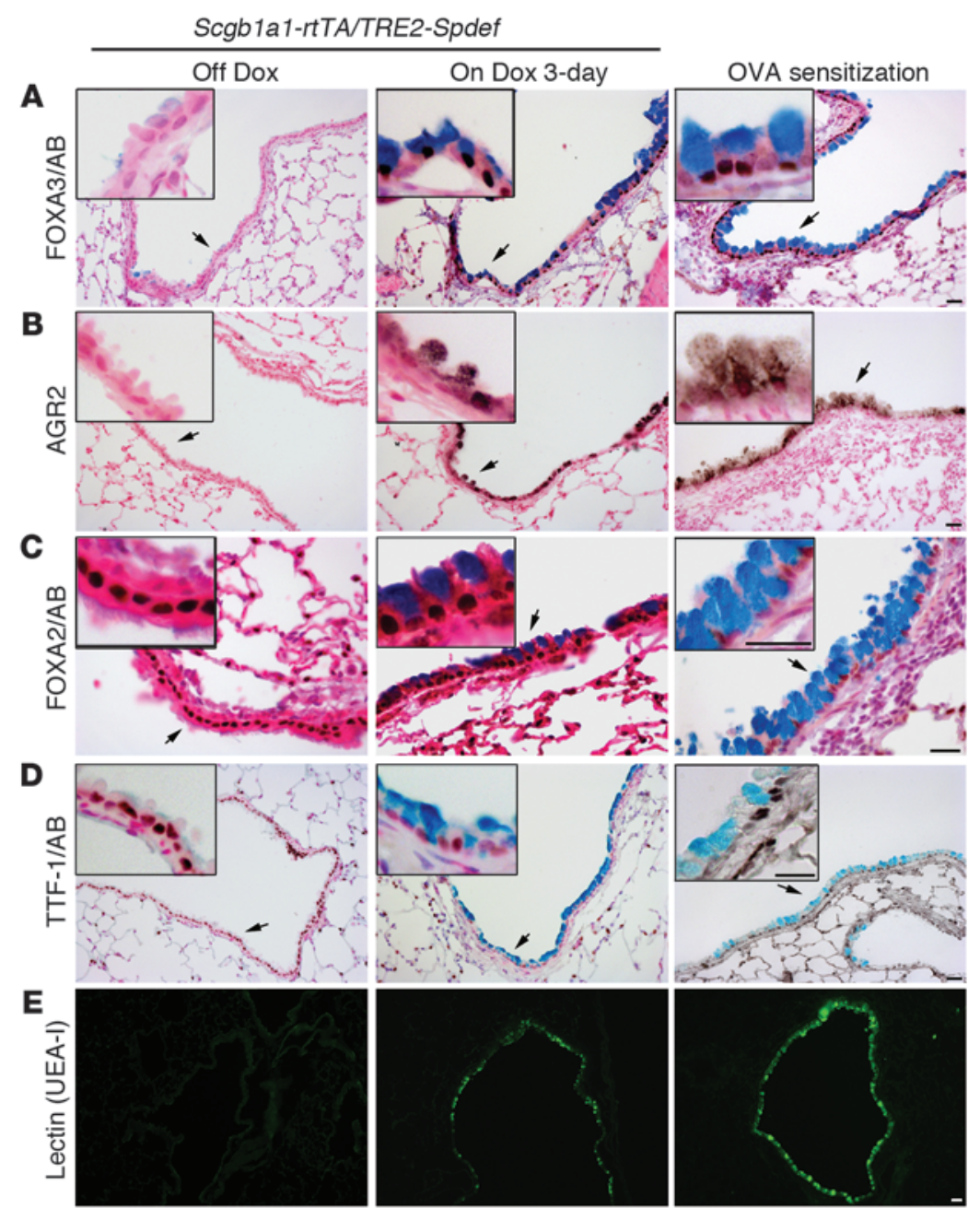

\section{Figure 3}

SPDEF or allergen sensitization induced FOXA3, AGR2, and UEA-I lectin staining and inhibited FOXA2 and TTF-1 staining in goblet cells in vivo. Expression of SPDEF in Scgb1a1-rtTA/TRE2-Spdef mice or intrapulmonary OVA sensitization induced FOXA3 (A), AGR2 (B), and UEA-I lectin (E) staining and decreased staining of FOXA2 (C) and TTF-1 (D) in goblet cells. Arrows indicate regions selected in the inserts. Scale bars: $25 \mu \mathrm{m}$.

Coexpression of SPDEF, FOXA3, and AGR2 in pulmonary diseases. Immunohistochemical staining for SPDEF, FOXA3, and AGR2 was assessed in bronchial tissues from patients with pulmonary diseases caused by CF or cigarette smoking, disorders in which goblet cell hyperplasia and mucus hypersecretion are prominent. Alcian blue, SPDEF, FOXA3, and AGR2 staining were markedly increased at sites of goblet cell hyperplasia and were not readily detected in normal airway epithelium (Figure 5). In contrast, SPDEF, FOXA3, and AGR2 were expressed in goblet cells in the normal submucosal glands in both humans and mice (Figure 5F and data not shown).

SPDEF is required for allergen-induced goblet cell differentiation. Spdef ${ }^{\prime-}$ mice breed and survive normally in the vivarium. Spdef ${ }^{/-}$, Spdef $f^{+-}$, and $S p d e f^{+/+}$mice were sensitized by repeated systemic injection followed by intrapulmonary administration of OVA. Lung histology was unaltered in Spdef ${ }^{/-}$mice as assessed by light microscopy; however, goblet cells were absent in the tracheal and laryngeal submucosal glands of Spdef ${ }^{\prime-}$ mice prior to allergen exposure (Figure 6). As in wild-type mice, OVA sensitization induced goblet cell differentiation in Spdeff/- mice as indicated by Alcian blue staining of acidic

Foxa2 mRNAs was confirmed by immunohistochemistry. Staining for FOXA2 and TTF-1 was markedly inhibited in goblet cells induced by SPDEF or after pulmonary OVA sensitization, while FOXA3 and AGR2 were induced (Figure 3, A-D). Consistent with the role of SPDEF in promoting mucin biosynthesis, protein glycosylation in the goblet cells induced by SPDEF or pulmonary OVA sensitization was detected by staining of the lectin Ulex europaeus agglutinin I (UEA-I), which recognized the L-fucose moiety of glycoproteins (30) (Figure 3E). The induction of AGR2 was of particular interest, since Agr2 encodes a potential chaperone required for mucin packaging in goblet cells in vivo (31). SPDEF was colocalized with FOXA3 and AGR2 in goblet cells in vivo, indicating that SPDEF and FOXA3 may cooperate in the regulation of gene expression (Figure 4, A and B). SPDEF and FOXA3 synergistically activated the Agr2-luciferase promoter and endogenous Agr2 mRNA expression in vitro (Figure 4, C and D). We conclude that SPDEF induces goblet cell differentiation, increasing the expression of genes associated with mucin biosynthesis, glycosylation, and packaging, while inhibiting genes characteristic of Clara cells in the normal bronchiolar epithelium, including genes regulating fluid and electrolyte transport as well as innate host defense. Since TTF-1 and FOXA2 are important transcriptional regulators of genes expressed selectively in Clara cells, their inhibition by SPDEF likely accounts for changes of gene expression associated with Clara cells. mucopolysaccharides and increased staining of SPDEF, FOXA3, MUC5AC, and UEA-I lectin (Figure 7). In contrast, neither goblet cell morphology nor the goblet cell-associated transcription factor FOXA3 was detected in the bronchial-bronchiolar epithelial cells in Spdef ${ }^{-1}$ mice before or after allergen sensitization. MUC5AC and staining for mucus-associated fucosyl glycoconjugates with UEA-I lectin were markedly inhibited but not absent in the Spdef ${ }^{\prime-}$ mice after allergen exposure (Figure 7). Pulmonary inflammation and eosinophilic and lymphocytic infiltration associated with allergen exposure were similar in Spdef ${ }^{+-}$and Spdef ${ }^{/-}$mice (Supplemental Figure 3). To assess whether expression of SPDEF in airway epithelial cells was sufficient to induce MUC5AC expression in vitro, NCI-H292 cells and HBEC 3KT (human bronchial epithelial cells) (32) were infected with lentivirus expressing the Spdef cDNA. SPDEF induced endogenous MUC5AC expression in both cell lines (Figure 8). SPDEF was induced by IL-13 in primary mouse tracheal epithelial cells grown at air-liquid interface (Supplemental Figure 4), consistent with the role for TH2 cytokine signaling in the regulation of SPDEF.

\section{Discussion}

The respiratory tract is continuously exposed to inhaled irritants, particles, and pathogens, necessitating the evolution of robust innate and acquired host defense systems to maintain pulmonary 

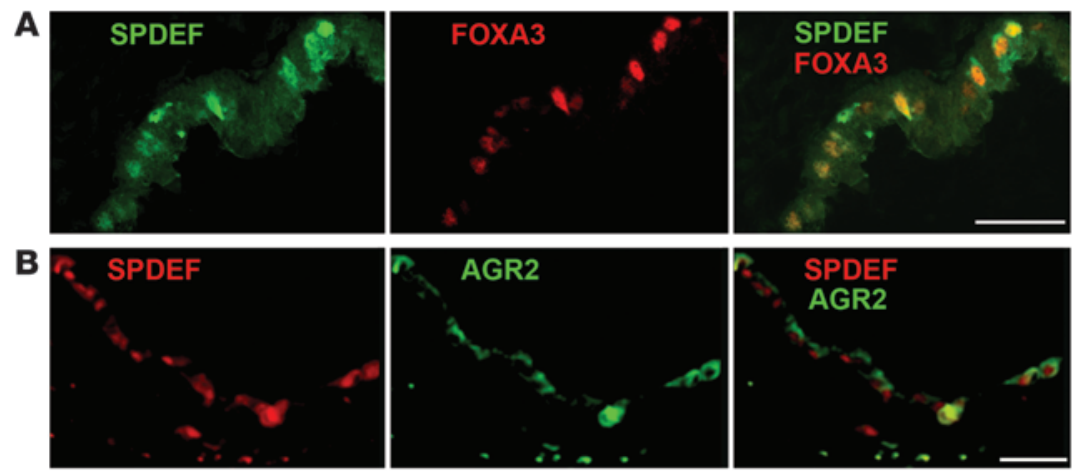

C

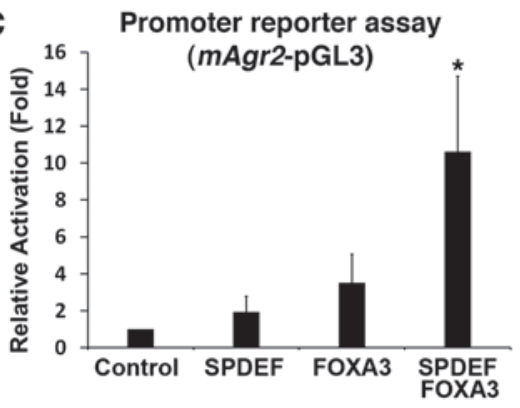

D

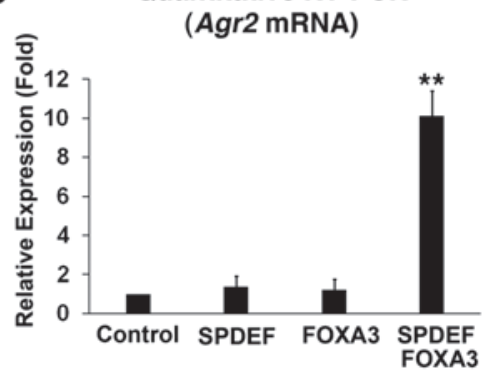

\section{Figure 4}

SPDEF and FOXA3 are coexpressed and induce Agr2 expression. Scgb1a1-rtTA/TRE2-Spdef transgenic mice were treated with doxycycline for 3 days to induce SPDEF in bronchiolar epithelial cells (A and $\mathbf{B})$. SPDEF was colocalized with FOXA3 in nuclei (A) and AGR2 in the cytoplasm (B) of goblet cells as assayed by immunofluorescence microscopy (yellow indicates colocalization of nuclear SPDEF and FOXA3). Luciferase reporter constructs containing the promoter region from the mouse Agr2 (1.6 kb) gene was transfected into primary sheep tracheal epithelial cells. Synergistic activation of Agr2 promoter was observed when SPDEF and FOXA3 were cotransfected (C). Endogenous Agr2 mRNA expression was induced by transfection with both SPDEF and FOXA3 expression plasmids in the mouse lung epithelial cell line MLE15 (D). Results were expressed as mean \pm SD of 3 independent experiments. ${ }^{*} P<0.02$; ${ }^{* *} P<0.01$ versus control constructs. Scale bars: $25 \mu \mathrm{m}$. sterility and homeostasis. Mucociliary clearance, the production of innate defense proteins, and the activity of the immune system all serve to maintain lung function after birth. The respiratory epithelium adapts to inflammatory stimuli, often dramatically increasing the number and activity of goblet cells. Goblet cell differentiation and excessive production of mucus are common features of many chronic pulmonary diseases, including asthma, $\mathrm{CF}$, and chronic obstructive pulmonary disease. The present study demonstrates that goblet cells induced by allergens or by the expression of SPDEF are derived by differentiation of existing Clara cells and not by proliferation. Thus, the findings support the concept that goblet cells formed in the present model occur by metaplasia rather than by "hyperplasia." SPDEF is required for goblet cell differentiation in normal tracheal-laryngeal submucosal glands and is required for goblet cell differentiation following allergen exposure. SPDEF regulates a group of genes associated with goblet cell differentiation, mucin biosynthesis, and secretion. SPDEF plays a central role in the regulation of goblet cell differentiation in epithelial cells in the respiratory tract, interacting with a transcriptional network that includes suppression of TTF-1 and FOXA2 and induction of FOXA3.

SPDEF was initially identified in prostate epithelium, where it interacts with NKX-3.1 in the regulation of prostate-specific antigen (PSA) (33). Spdef mRNA and protein are expressed in a number of organs that are enriched in epithelial cells of mucus and other secretory lineages, including breast, pancreas, and both reproductive and gastrointestinal tracts, supporting its potential role in goblet cell differentiation. In the intestine, secretory cell (goblet and Paneth cells) differentiation is regulated by the Notch signaling pathway $(7,8)$ and transcription factors including MATH1 (also termed atonal homolog 1 [ATOH1], Drosophila) (34) and growth factor independent 1 (GFI1) (35), while in the colon, KLF4 regulates goblet cell differentiation (36). Loss of MATH1/ATOH1 caused loss of GFI1, a zinc-finger transcription factor required for intestinal secretory lineage specification, which in turn decreased
Spdef mRNA and reduced goblet cell number (35). Thus, expression of Spdef in postmitotic intestinal epithelial cells is regulated by the Notch signaling pathway and its downstream transcription factors MATH1/ATOH1 and GFI1, which are required for secretory lineage specification. However, it is still unclear that expression of SPDEF is regulated by Notch in the mature respiratory epithelium. Notch signaling pathway has been recently reported to promote airway goblet cell differentiation in a STAT6-independent manner in fetal tracheal explants in vitro (37). In the adult respiratory epithelium, SPDEF expression and goblet cell differentiation were induced by Th2 cytokines in a STAT6-dependent manner in vivo $(10,16)$. In the lung, GFI1 expression is confined to pulmonary neuroendocrine cells (38), and Klf4 mRNA was not induced in association with goblet cell differentiation in the pulmonary allergy and IL-13 microarray databases presently analyzed, suggesting that at least some aspects of the transcriptional regulation of goblet cell differentiation may be tissue specific. Nevertheless, SPDEF is expressed in many tissues containing goblet cells, including the respiratory and gastrointestinal epithelium $(16,39)$.

The present data support potential roles of FOXA2, TTF-1, and FOXA3 in a transcriptional network associated with goblet cell differentiation or function (Figure 9). SPDEF inhibited FOXA2 and TTF-1 and induced FOXA3, the latter a transcription factor that is not abundantly expressed in the normal lung (40). We observed reduced expression of FOXA2 and increased expression of SPDEF and FOXA3 during goblet cell differentiation caused by OVA sensitization and in lung tissue from patients with CF and chronic smoking. FOXA2 and FOXA3 staining were mutually exclusive following expression of SPDEF or its induction by allergen. Their colocalization in goblet cells and the finding that SPDEF and FOXA3 synergistically induced Agr2 indicate their potential roles in goblet cell differentiation.

$S P D E F$ regulates expression of genes regulating mucin biosynthesis. The mRNA microarray analysis of SPDEF-induced genes in the conducting airways indicated that the expression of genes associated 
A Bronchiolitits obilterans
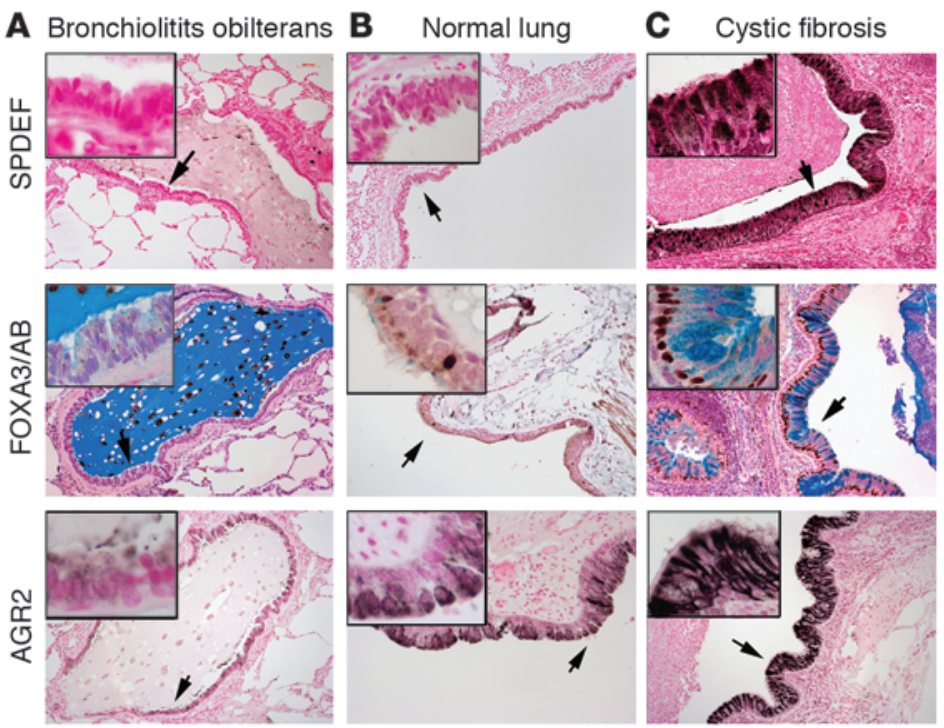

D
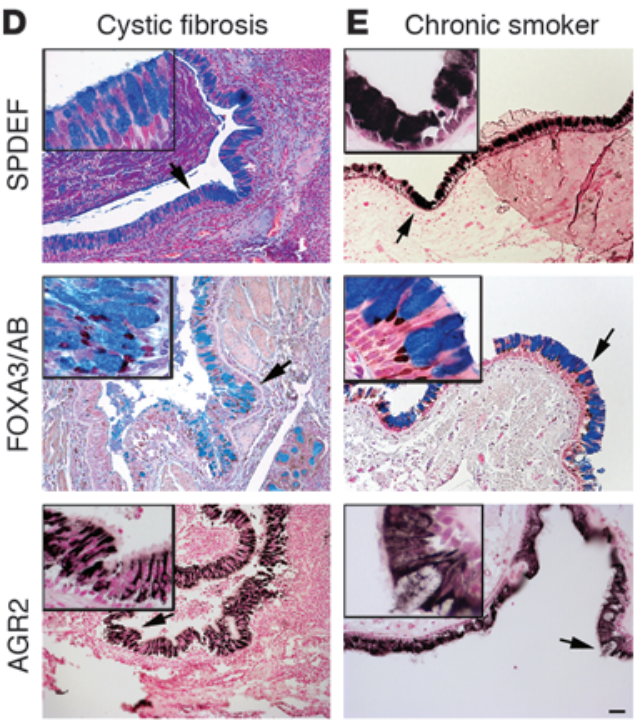

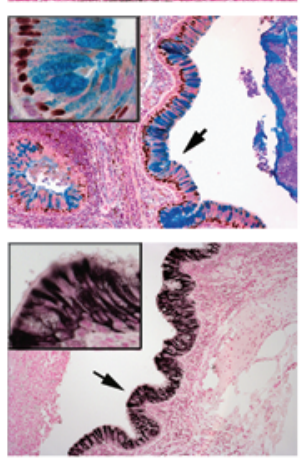

Normal
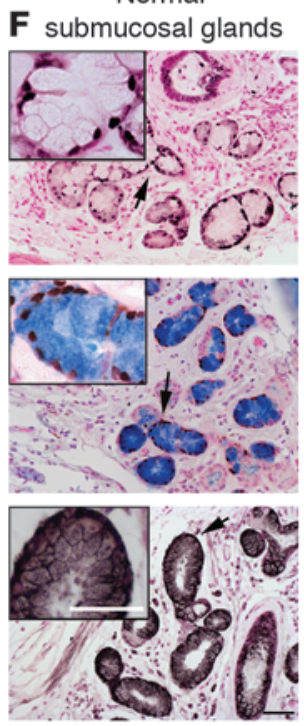

Figure 5

SPDEF, FOXA3, and AGR2 in human lung tissue. SPDEF, FOXA3, and AGR2 were detected by immunostaining of human lung tissue. Tissue from a pediatric patient with bronchiolitis obliterans lacked goblet cells; SPDEF, FOXA3, and AGR2 staining were absent (A). In normal lung, SPDEF staining was not detected and FOXA3 staining was observed in relatively few bronchial epithelial cells in regions lacking goblet cells, where AGR2 was weakly expressed (B, $n=3)$. Intense SPDEF staining was seen in bronchial tissue from patients with CF $(n=5)$, particularly in regions of goblet cell hyperplasia (top panel of C), as indicated on adjacent section stained with Alcian blue (top panel of $\mathbf{D}$ ) and in tissue from patients with history of chronic smoking (top panel of E). FOXA3 staining was also increased in the nuclei of goblet cells from lung tissues of CF patients $(n=4)$ and patients with history of chronic smoking (middle panels of $\mathbf{C}, \mathbf{D}$ and $\mathbf{E}$, respectively). AGR2 was increased in bronchial epithelial cells of CF patients' and smokers' lungs (lower panels of $\mathbf{C}, \mathbf{D}$, and $\mathbf{E}$, respectively). SPDEF, FOXA3, and AGR2 were detected in mucous cells of bronchial submucosal glands of normal human lung $(\mathbf{F}, n=3)$ as well as in smokers' lungs (data not shown). Arrows indicate regions selected in the inserts. Scale bars: $25 \mu \mathrm{m}$.

Mucin biosynthesis begins with the attachment of $N$-acetylgalactosamine (GalNAc) to a serine or threonine residue, a process catalyzed by various UDP-GalNAc:polypeptide (where UDP indicates uridine diphosphate) GalNAc transferases (pGalNAc-T subfamily) (42). The synthesis of more complex, functional mucin carbohydrate requires the formation of core $2 \beta 1,6$ branched structure in the mucin glycan chain by the $\beta-1,6-N$-acetylglucosaminyltransferase family of enzymes (also known as the C2GnT family) (43). mRNAs of all 3 members in the C2GnT family that mediate production of functional mucins (Gcnt1, -2, and -3) were significantly induced by SPDEF (increased 3.4-, 1.8-, and 9.3-fold respectively), strongly supporting its regulatory role in mucin biosynthesis and secretion. Among these enzymes, GCNT3 was induced the most by the expression of SPDEF in the airway epithelium (Figure 3E). GCNT3 mediates both core 2 and core $4 O$-glycan branching, 2 important steps in mucin-type biosynthesis (44). with mucin biosynthesis was induced most dramatically. Mucins are large molecular weight glycoproteins with $10 \%$ to $20 \%$ protein and $80 \%$ to $90 \%$ carbohydrate components. Biosynthesis of mature glycosylated mucin requires (a) transcription of a $M U C$ gene to encode a MUC mRNA in nucleus, (b) translation into a MUC protein backbone on a ribosome that is inserted into the ER, a cellular compartment for protein folding, and (c) posttranslational modification of mucin core proteins by one or several glycosyltransferases in the Golgi. In the present study, mRNA microarray analysis supports the concept that SPDEF influences mucus production at least in part by regulation of AGR2, a mucin chaperone protein in the ER and peptidyl $\mathrm{N}$-acetylgalactosaminyl transferases in the Golgi. AGR2 was identified as an ER protein that belongs to the protein disulfide isomerase family (PDI) of chaperones, which facilitate the folding of proteins targeted for the secretory pathway (41). AGR2 binds to unfolded parts of mucin protein in the ER to enhance protein folding and the posttranslational modification of serine and threonine residues, the sites of $O$-glycosylation in Golgi (31).
Gcnt3 mRNA is expressed primarily in mucus-secreting tissues, including the gastrointestinal tract and trachea, and is induced by retinoic acid, TNF- $\alpha$, and IL-13 $(45,46)$. Previous studies demonstrated that GCNT1 and 3 enzyme activities and mRNA levels were induced in airway epithelial cells following exposure to Th2 cytokines (IL-4 and IL-13) in a process mediated by the JAK-STAT signaling pathway $(23,45)$. Consistent with the important role of SPDEF in the regulation of mucin glycosylation, staining for UEA-I lectin was induced by SPDEF.

In the present study, goblet cell differentiation was associated with the increased expression of SPDEF and FOXA3 in bronchiolar epithelial cells, consistent with the microarray results showing the induction of many asthma-related and IL-13-responsive genes. $M u c 5 a c$, encoding a major mucin type secreted from airway goblet cells, was induced by SPDEF expression in vitro and in vivo. Expression of SPDEF was sufficient to induce MUC5AC in H292 and HBEC cells in vitro. Muc16 mRNA was markedly induced by SPDEF in vivo. MUC16 expression in tracheal epithelium, submucosal glands, and cultured bronchial epithelial cells was recently 


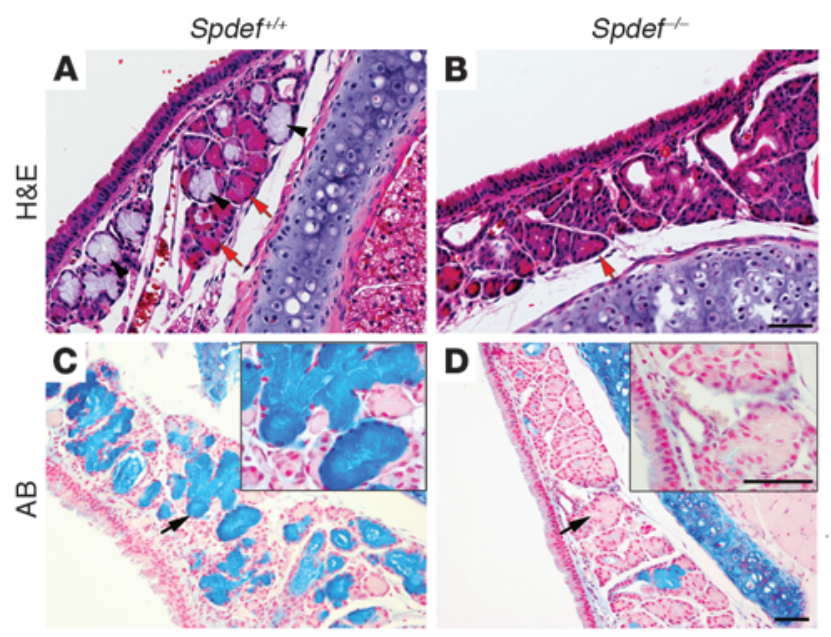

reported (17). These findings indicate that SPDEF regulates a number of genes involved in mucin synthesis, its glycosylation, and packaging. It remains unclear whether the effects of SPDEF represent direct cell autonomous effects on target gene expression or more complex effects on other regulators of goblet cell differentiation and function.

Expression of SPDEF in Clara cells caused their differentiation into goblet cells and inhibited a number of genes regulating host defense as well as fluid and electrolyte transport that are normally expressed in Clara cells and are known transcriptional targets of FOXA2 and TTF-1. This inhibitory effect is mediated in part by loss of FOXA2 and TTF-1 expression caused by induction of SPDEF in the airway epithelium. It is presently unclear whether this inhibition is mediated by direct or indirect effects of SPDEF on these transcriptional targets (Figure 9).

SPDEF, FOXA3, and AGR2 were increased in both the mouse models and in human lung tissue wherein their expression was associated with the presence of goblet cells, supporting the conservation of the proposed regulatory network. Since the cellular composition of the mouse airway differs from that of the human, the cellular origin of goblet cells in the human airway in which basal rather than Clara cells are more prominent, remains unclear.

Pulmonary allergen exposure and chronic inflammatory diseases of the lung are associated with infiltration by many cell types and the expression of numerous cytokines, chemokines, and other inflammatory mediators. The present findings indicate that these complex signals influence goblet cell differentiation in the respiratory epithelium via the transcription factor SPDEF and its influence on an extended transcriptional network (Figure 9). Changes in gene expression, cell differentiation, and morphology caused by SPDEF occur rapidly and reversibly without the activation of cell proliferation. Goblet cell differentiation and mucin secretion also occur following acute inflammation. Thus, SPDEF plays a central role in the regulation of a gene network that responds to pathogens or toxicants, in turn changing epithelial cell differentiation and mucociliary clearance that together play a role in innate host defense of the lung. Such changes in cell differentiation and function may represent an adaptive change in the epithelium that occurs without cell death, minimizing the need to activate cell proliferation. Since mucus hyperproduction contributes to the pathogenesis of acute and chronic pulmonary disorders, knowledge regarding the regulation and function of SPDEF in the respiratory

\section{Figure 6}

Absence of mucous (goblet) cells in tracheal and laryngeal submucosal glands in Spdef-- mice. Tracheal/laryngeal glands in wild-type mouse are shown after H\&E and Alcian blue staining. Distinct mucous cells (black arrowheads in A) and serous cells (red arrows in $\mathbf{A}$ and B) from wild-type mice are shown. Mucous cells were not detected in the submucosal glands of Spdef/- mice, although serous glands were present (B). Alcian blue staining was readily detected in submucosal glands of wild-type mice (arrow) (C) but rarely observed in submucosal glands of Spdef $f^{-}$mice (arrow) (D). Arrows indicate regions of higher magnification shown in inserts ( $\mathbf{C}$ and $\mathbf{D}$ ). Figures are representative of $n=3$ individual mice of each genotype. Scale bars: $50 \mu \mathrm{m}$.

tract provides a framework for the development of new strategies for diagnosis and therapy for chronic lung diseases.

\section{Methods}

Mouse models, OVA sensitization, and BrdU administration. Mouse strains included in this study were Spdef $/$ - mice produced in the laboratory of Hans Clevers, Netherlands Institute of Developmental Biology. Young adults of approximately 6 to 8 weeks of age were utilized in most studies. Scgb1a1$r t T A$ (line 2) (47)/TRE2-Spdef (16) mice in the FVB/N strain were treated with doxycycline $(625 \mathrm{mg} / \mathrm{kg}$ of food). R26R mice, kindly provided by P. Soriano (Fred Hutchinson Cancer Research Center, Seattle, Washington, USA) (48), were bred to Scgb1a1-rtTA (line 2) (Otet) $>C M V$-Cre mice for lineage tracing (Figure 1A). The OVA sensitization protocol was described previously (14). Wild-type FVB/N mice were obtained from The Jackson Laboratory. To induce pulmonary goblet cell hyperplasia, mice received i.p. injections with $100 \mu \mathrm{g}$ OVA (grade V; Sigma-Aldrich) and $1 \mathrm{mg}$ Imject Alum (Thermo Scientific) as adjuvant, followed by 2 i.n. instillations of $50 \mu \mathrm{g}$ of OVA or saline 3 days apart, starting at least 10 days after the second sensitization. BrdU (B5002; Sigma-Aldrich) was administered daily by i.p. injection $(50 \mu \mathrm{g} / \mathrm{g}$ body weight at a concentration of $10 \mathrm{mg} / \mathrm{ml}$ in sterile saline) during treatment with doxycycline (over 3 days) and nasal sensitization with OVA (day 24 through day 29; Figure 1B). Animal protocols were approved by the Cincinnati Children's Hospital Medical Center Institutional Animal Care and Use Committee in accordance with NIH guidelines.

Laser capture, RNA purification, and amplification. LCM was performed as described (49). To induce Spdef expression, 8-week-old adult male Scgb1a1-rtTA/TRE2-Spdef transgenic mice were treated with doxycycline for 3 days $(n=3)$. The control mice were the same age, sex, and genotype but were not treated with doxycycline $(n=3)$. Mice were anesthetized and exsanguinated, and the lungs were inflated with OCT (Fisher Scientific)/DEPC-PBS (where DEPC indicates diethylpyrocarbonate) with $10 \%$ sucrose $(50 \% \mathrm{v} / \mathrm{v})$ (sucrose, S0389; Sigma-Aldrich). After inflation, lungs were dissected, and lobes were separated, embedded in OCT, and snap-frozen in isopentane, and stored at $-80^{\circ} \mathrm{C}$. Tissue was sectioned at $-20^{\circ} \mathrm{C}$ in the cryostat. Thin sections $(10 \mu \mathrm{m})$ were collected on $1: 20$ poly-L-lysine-coated (P8920; Sigma-Aldrich) slides and stored at $-80^{\circ} \mathrm{C}$. Prior to laser capture microscopy, slides were fixed in iced DEPC $70 \%$ ethanol, washed in DEPC- $\mathrm{H}_{2} \mathrm{O}$, dehydrated in $95 \%$ and $100 \%$ ethanol, xylene, and air dried. Bronchiolar cells were captured by Veritas Microdissection Instrument (Model 704; Molecular Devices) with a laser set at 15 $\mu \mathrm{m}$. Total RNAs were purified by Arcturus PicoPure RNA Isolation Kit (Molecular Devices). RNAs were then subjected to 2 rounds of amplifica- 


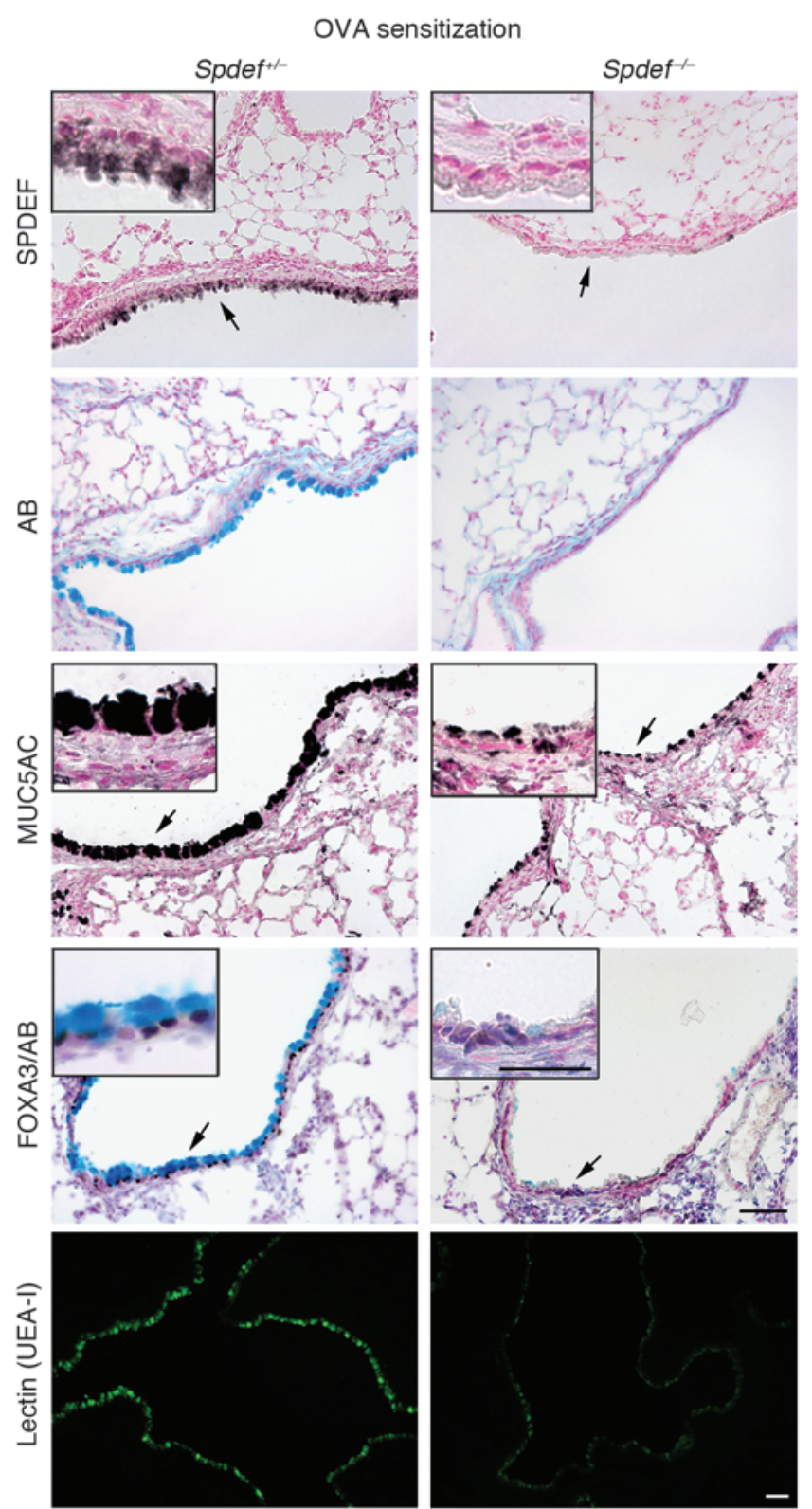

tion using TargetAmp 2-Round Aminoallyl-aRNA Amplification Kit 1.0 (EPICENTRE Biotechnologies).

RNA microarray analysis. The RNAs were then hybridized to the murine genome MOE430 chips (consisting of approximately 45,000 gene entries; Affymetrix) according to the manufacturer's protocol. The RNA quality and quantity assessment, probe preparation, labeling, hybridization, and image scan were carried out in the CCHMC Affymetrix Core using standard procedures. Affymetrix Microarray Suite 5.0 was used to scan and quantitate the gene chips under default scan settings. Hybridization data were subjected sequentially to normalization, transformation, filtration, and functional classification and pathway analysis as previously described $(50,51)$. Data analysis was performed with BRB Array Tools software package (http://linus.nci.nih.gov/BRB-ArrayTools.html). Genes differentially expressed in response to doxycycline treatment groups were identified using a random variance $t$ test, which is an improvement over the standard $t$ test, as its variance estimates are made on all genes with the same

\section{Figure 7}

SPDEF is required for goblet cell differentiation following intrapulmonary allergen sensitization. After intrapulmonary sensitization with OVA (as described in Figure 1), SPDEF was readily detected in the bronchiolar epithelial cells in Spdeft/- but not in Spdef ${ }^{--}$mice. The increased Alcian blue, MUC5AC, FOXA3, and UEA-I lectin staining in goblet cells after OVA sensitization seen in Spdef+/- mice was markedly inhibited in the Spdef-/ littermates. Arrows indicate regions selected in the inserts. Scale bars: $50 \mu \mathrm{m}$. Figures are representative of $n=4$ mice of each genotype.

treatment without assuming that all genes have the same variance (52). Gene expression changes were considered statistically significant if their $P$ values were less than 0.01 and fold changes were greater than 1.5 . We also performed a permutation test to provide a $90 \%$ confidence level that the false discovery rate was less than $10 \%$. The false discovery rate is the proportion of the list of genes claimed to be differentially expressed that are false positives. In addition, Affymetrix Present Call in at least 2 of 3 replicates and coefficient of variation among replicates of $50 \%$ or less were set as a requirement for gene selection.

Functional classification and pathway analysis. Gene Ontology Analysis was performed using the publicly available web-based tool DAVID (database for annotation, visualization, and integrated discovery). Gene sets associated with known pathways and disease states from Kyoto Encyclopedia of Genes and Genomes (KEGG) (http://www.genome.ad.jp/kegg/), GenMAPP (http://www.genmapp.org), and GEArrays (http://www.sabiosciences.com/) were identified by comparing the overlap of pathway genes with the differentially expressed genes and all genes in the MOE430 mouse genome. A gene ontology term is considered to be overly represented when a Fisher's exact test $P$ value is less than or equal to 0.01 and gene hits are equal to 10 or more. Potential protein/protein or protein/DNA interactions were identified using Ingenuity Pathway Analysis (Ingenuity). Genetic networks preferentially enriched for input genes were generated based on their connectivity. Statistical scores were calculated to rank the resulting networks and pathways using Fisher's right-tailed exact test. The score indicates the degree of relevance of a network to the input gene set, which takes into account the number of network-eligible genes and the size of the network.

Human specimens. Anonymous, deidentified, human adult and pediatric lung samples were obtained through the Department of Pathology, University of Cincinnati College of Medicine, and the Division of Pathology, Cincinnati Children's Hospital Medical Center, in accordance with institutional guidelines for use of human tissue for research purposes (courtesy of Gail Deutsch, Kathryn Wikenheiser-Brokamp, and Robert Baughman).

Immunohistochemistry, immunofluorescence, $X$-gal, and Alcian blue staining. Adult mouse lung was inflation fixed, embedded, sectioned, and immunostained. Alcian blue and immunohistochemical staining for SPDEF, CCSP, and phosphohistone $\mathrm{H} 3$ followed previously described methods (16). TTF-1 was detected by a mouse monoclonal TTF-1 antibody (8G7G3/1) as previously described (53). AGR2, MUC5AC (ab47044 and ab3649, respectively; Abcam), FOXA3 (SC-5361; Santa Cruz Biotechnology Inc.), BrdU (Zymed BrdU staining Kit; Invitrogen), and UEA-I (L9006; Sigma-Aldrich) staining followed standard procedures, as recommended by the manufacturers. Rat anti-mouse CD68 antibody (AbD Serotec) was used to detect monocytes and macrophages on frozen sections. Human SPDEF antibody (54) used on all human specimens was kindly provided by Dennis Watson (Medical University of South Carolina, Charleston, South Carolina, USA). Immunohistochemistry was performed using human SPDEF antibody at a dilution of 1:500 after antigen retrieval with citrate buffer and heat. Major basic protein (MBP) antibody was kindly provided by Jamie and Nancy Lee (Mayo Clinic, Scottsdale, Arizona, USA). 

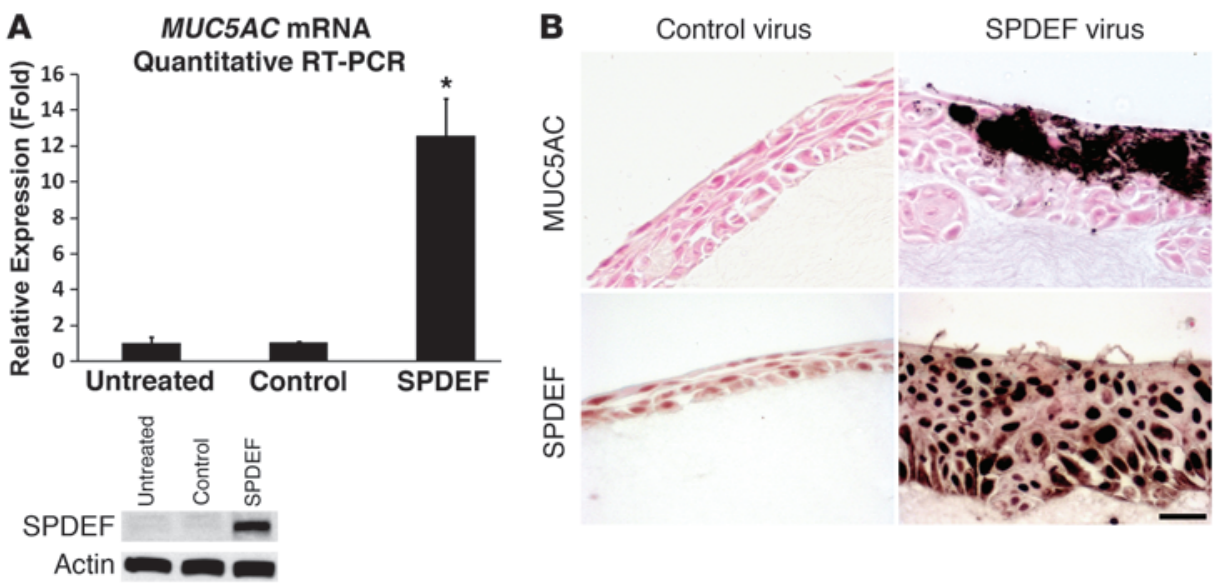

Figure 8

SPDEF-induced MUC5AC mRNA and protein expression. (A) SPDEF-induced MUC5AC mRNA expression in NCl-H292 cells in vitro. Lentivirus that expressed mouse SPDEF or GFP (control) protein was used to infect $\mathrm{H} 292$ cells. After 5 days, MUC5AC mRNA was increased approximately 12-fold in the cells expressing SPDEF compared with uninfected cells or those expressing GFP. Expression of SPDEF is shown by Western blot in the lower panel. Graphs express the mean \pm SD of 3 independent experiments. ${ }^{*} P<0.01$ versus control virus. (B) SPDEF-induced MUC5AC expression in HBECs. Cells were infected with lentivirus expressing SPDEF or control virus (same as in A) followed by culture at airliquid interface for 4 weeks and assayed by immunohistochemistry. MUC5AC was induced by expression of SPDEF. Figures are representative of 3 independent experiments. Scale bar: $25 \mu \mathrm{m}$.

To detect $\beta$-gal expression, lungs were inflation fixed with $2 \%$ PFA fixative for 10 hours at $4^{\circ} \mathrm{C}$ and then processed for preparation of frozen sections. $\mathrm{X}$-gal enzymatic reaction was performed by incubating the lung sections with $5 \mathrm{mM} \mathrm{K}_{4} \mathrm{Fe}(\mathrm{CN})_{6}, 5 \mathrm{mM} \mathrm{K}_{3}(\mathrm{CN})_{6}$, and $1 \mathrm{mg} / \mathrm{ml} \mathrm{X}$-gal in PBS (pH 7.2) at $30^{\circ} \mathrm{C}$ for $4-8$ hours. After $\mathrm{X}$-gal staining, the same slides were subjected to immunofluorescence staining for CCSP and FOXJ1 following previously described methods (16). To identify goblet cells, Alcian blue stain- ing was performed after immunohistochemical staining, using a 3-minute incubation in $3 \%$ acetic acid and a 10-minute incubation in $1 \%$ Alcian blue (Poly Scientific). Then slides were rinsed with running water for 5 minutes followed by 2 minutes in nuclear fast red, dehydration, and coverslipping with mounting media.

Plasmids and cell lines. Promoter regions were selected based on the sequence similarity and the conservation of the predicted transcription factor bind-

\section{Figure 9}

Schematic representation of genomic responses induced by conditional expression of SPDEF in the airway epithelium. SPDEF promotes goblet cell differentiation and mucus production while suppressing expression of genes associated with Clara cells. SPDEF interacts in a regulatory network mediated in part by the inhibition of FOXA2 and TTF-1 and the induction of FOXA3. SPDEF is induced, while FOXA2 is inhibited by pulmonary allergen or IL-13 in a STAT6-dependent manner (A). SPDEF induced the expression of a number of genes regulating mucin biosynthesis, particularly mucin glycosylation (B) and goblet cell differentiation (C) while suppressing those regulating fluid and electrolyte transport and innate host defense in part by its inhibitory effects on TTF-1 and FOXA2 transcription factors that control differentiation and function of the normal bronchiolar epithelium (D). The network indicates a regulatory relationship but does not imply direct transcriptional control of each gene by SPDEF or other transcription factors.

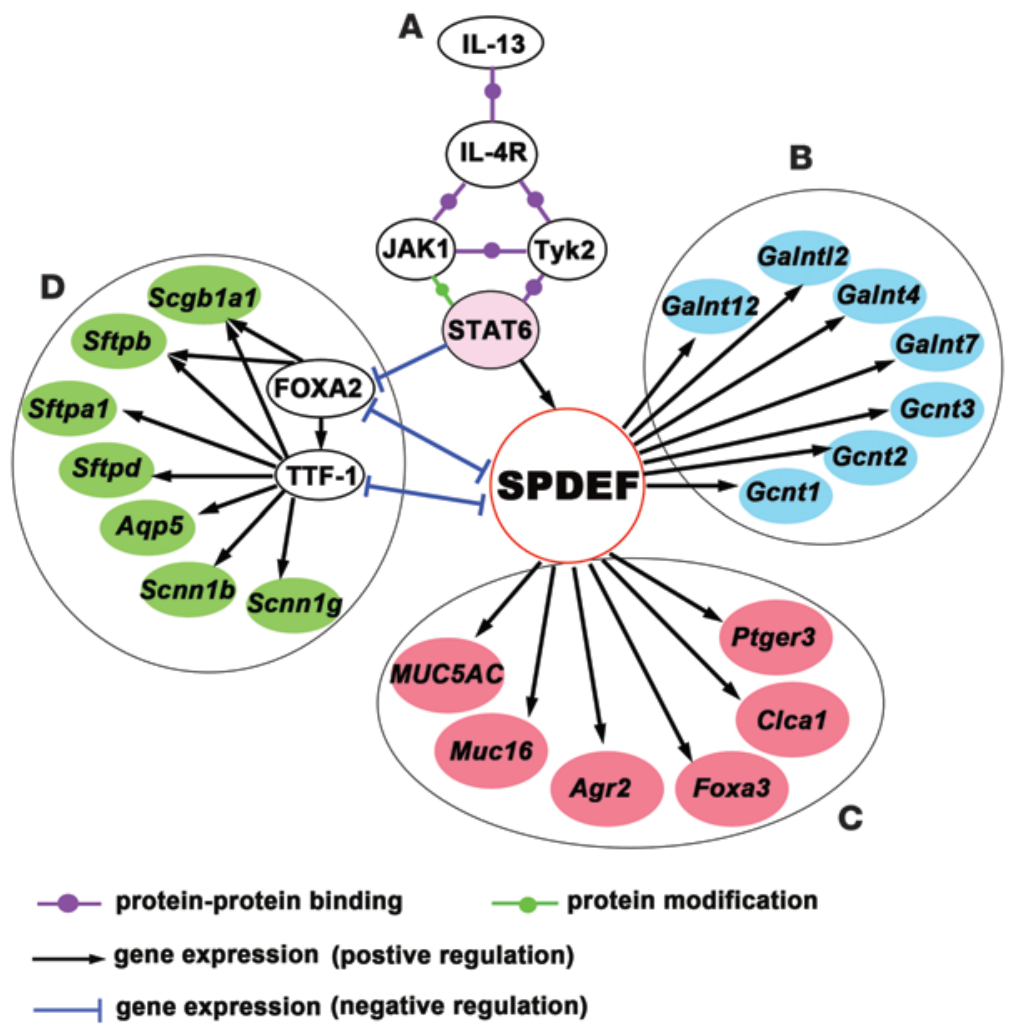


ing sites present in human, mouse, and rat genomes. All the PCR reactions were performed using GC-Rich PCR system (Roche) and then cloned into TA cloning vector pSC-A (StrataClone PCR cloning kit; Stratagene) for sequencing. The primer sets for cloning human FOXA3 3-kb promoter were as follows: 5'-GCTCGAGCCTGCAGGAGCTAGATTTTATGC-3' and $5^{\prime}$-AtCTCGAGTCTGGATCTCTCAGCGGGCACGG-3'. The PCR product was cloned into PSC-A vector, isolated by cutting with HindIII and SpeI, and cloned into HindIII and NheI sites of the pGL3 basic vector (Promega). The primer sets for cloning the mouse Agr2 1.6-kb promoter were as follows: $5^{\prime}$-TTCTCGAGAATGGGTGGGATTTCGGGTC-3' and 5'-ATCTCGAGTGCTTGTCAATTGCCTTACC-3'. Those for mouse Muc16 $2.6 \mathrm{~kb}$ promoter were as follows: $5^{\prime}$-TTCTCGAGTACTCCACTTATAAATGAG-3' and 5'-TTCTCGAGGAAAACTCATATCATAAGC-3'. The PCR fragments were then cloned into the XhoI site of PGL3 basic vector. The FOXA3 expression vector was made by amplifying the mouse 1-kb Foxa3 cDNA using the primer sets 5'-TTGGATCCATGCTGGGCTCAGTGAAGATG-3' and $5^{\prime}$-TGGATCCCTAGGATGCATTAAGCAGAGAGCG-3' and subcloned into the BamHI site of pcDNA5/TO (Invitrogen). SPDEF expression vector was made by cloning 1-kb Spdef cDNA from TRE-Spdef (16) plasmid using the primer sets $5^{\prime}$-AATTCTAGAGATGGGCAGTGCCAGCCCAGG-3' and 5'-ATTCTAGATCAGACTGGATGCACAAATT-3' and subcloned into the XbaI site of pcDNA5/TO. To make SPDEF-expressing lentivirus, Spdef cDNA was cloned into $\mathrm{p} 3 \times$ FLAG-myc-CMV-26 expression vector (E6401; Sigma-Aldrich) and cut out from SacI and BamHI to make FLAG-Spdef-myc fusion protein fragment. This fragment was inserted into the BamHI site of PGK-IRES-EGFP (where PGK indicates phosphoglycerate kinase and IRES indicates internal ribosome entry site) backbone modified from a previously described lentiviral vector (55) via blunt-end ligation to make the plasmid that was packaged to generate the SPDEF-expressing lentivirus. The control virus was made by cutting out the FLAG-myc fragment from the p3 $\times$ FLAG-myc-CMV-26 expression vector and cloned into the PGK-IRESEGFP backbone with the same cloning strategy to make the SPDEF virus. Air-liquid interface culture of mouse tracheal epithelial cells was performed as previously described (56). The mouse IL-13 used for in vitro culture was purchased from R\&D Systems.

$3 D$ air-liquid interface culture of the HBECs and culture of NCI-H292 cells. HBECs were stably infected with either control virus or SPDEF-expressing virus. The $3 \mathrm{D}$ cultures were established as previously described (57). The HBECs were seeded in growth medium on top of the gels. When cultures became confluent in submerged conditions, they were switched to differentiation medium (58) with few alterations ( $2 \mu \mathrm{M}$ hydrocortisone, $2 \mu \mathrm{g} / \mu \mathrm{l}$ transferrin, $0.55 \mu \mathrm{M}$ epinephrine, $25 \mathrm{mg}$ bovine pituitary extract) for 2 days. The cultures were then raised to an air-liquid interface and grown for 4 weeks. The cells were then fixed in $4 \%$ paraformaldehyde $(20$ $\mathrm{mM}$ Tris- $\mathrm{HCl}, \mathrm{pH} 7.6,137 \mathrm{mM} \mathrm{NaCl}$ ), processed, embedded in paraffin, and prepared for immunohistochemistry. Human bronchial epithelial $\mathrm{NCl}-\mathrm{H} 292$ cells obtained from ATCC were grown in RPMI supplemented with $10 \%$ fetal calf serum and the antibiotics penicillin/streptomycin. Cells at $30 \%$ confluence in 6-well plates were infected with either control virus or SPDEF-expressing virus. Five days after infection, cells were harvested for mRNA (MUC5AC) and protein (SPDEF and actin) analysis.

Promoter reporter assays. Promoter reporter constructs were cotransfected into primary sheep tracheal epithelial cells (aSTEPC) with CMV- $\beta$-gal plasmid (Clontech) and/or transcription factor expression plasmid using Lipofectamine 2000 (Invitrogen) as previously described (16). Growth medium was changed into a differentiation medium (MTEC/Nu, mouse tracheal epithelial cells culture medium, $2 \% \mathrm{Nu}$ serum) (56) after transfection. Cell lysates were collected for luciferase activity assay 24 hours after transfection. All transfection assays were performed with primary sheep adult tracheal epithelial cells at passage 3 or 4 . Relative promoter activities were normalized to internal control, the $\beta$-gal activity, and shown as mean \pm SD.

Quantitative RT-PCR and statistics. Total RNAs obtained from LCM of bronchiolar tissue were reverse transcribed to cDNA by Verso cDNA Kit (Thermo Scientific). Quantitative RT-PCR was performed using Taqman probes and primer sets (Applied Biosystems) specific for Spdef (assay ID Mm00600221_m1), Muc16 (assay ID Mm01177119_g1), Ptger3 (assay ID Mm01316856_m1), Clca1 (assay ID Mm00777368_m1), Agr2 (assay ID Mm00507853_m1), Gcnt3 (assay ID Mm00511233_m1), and MUC5AC (assay ID Hs01365616_m1). A probe and primer set for ribosomal 18S (part number 4352930E) was used as normalization control. PCR reactions were performed by using $25 \mathrm{ng}$ cDNA per reaction in a 7300 realtime PCR System (Applied Biosystems). The quantification RT-PCR data to confirm microarray results were analyzed by Hsu's MCB (best) test (59). Other quantification data were analyzed by 2 -tailed, type 1 Student's $t$ test in this study; $P<0.05$ was considered significant.

\section{Acknowledgments}

The authors acknowledge support from Ann Maher for preparation of the manuscript; the Morphology Core in the Division of Pulmonary Biology at Cincinnati Children's Hospital Medical Center; Michael Mucenski, Michael Bruno, and Angela Keiser for technical support; and Johannes C.M. van der Loo, Punam Malik, and Anusha Sridharan for assistance with lentiviral vectors. Grant support was provided by the NIH to J.A. Whitsett (HL090156 and HL095580) and T.R. Korfhagen (HL095580).

Received for publication May 4, 2009, and accepted in revised form July 15, 2009.

Address correspondence to: Jeffrey A. Whitsett, Division of Pulmonary Biology, Cincinnati Children's Hospital Medical Center, MLC 7029, 3333 Burnet Avenue, Cincinnati, Ohio 452293039, USA. Phone: (513) 803-2790; Fax: (513) 636-7868; E-mail: jeff.whitsett@cchmc.org.
1. Perl, A.K., Wert, S.E., Nagy, A., Lobe, C.G., and Whitsett, J.A. 2002. Early restriction of peripheral and proximal cell lineages during formation of the lung. Proc. Natl. Acad. Sci. U. S. A. 99:10482-10487.

2. Rogers, D.F. 1994. Airway goblet cells: responsive and adaptable front-line defenders. Eur. Respir. J. 7:1690-1706

3. Rose, M.C., and Voynow, J.A. 2006. Respiratory tract mucin genes and mucin glycoproteins in health and disease. Physiol. Rev. 86:245-278.

4. Maeda, Y., Dave, V., and Whitsett, J.A. 2007. Transcriptional control of lung morphogenesis. Physiol. Rev. 87:219-244.

5. Ishikawa, N., Horii, Y., Oinuma, T., Suganuma, T., and Nawa, Y. 1994. Goblet cell mucins as the selective barrier for the intestinal helminths:
T-cell-independent alteration of goblet cell mucins by immunologically "damaged" Nippostrongylus brasiliensis worms and its significance on the challenge infection with homologous and heterologous parasites. Immunology. 81:480-486.

6. Salathe, M., Forteza, R., and Conner, G.E. 2002. Post-secretory fate of host defence components in mucus. Novartis Found. Symp. 248:20-26; discussion 27-37, 277-282.

7. Zecchini, V., Domaschenz, R., Winton, D., and Jones, P. 2005. Notch signaling regulates the differentiation of post-mitotic intestinal epithelial cells. Genes Dev. 19:1686-1691.

8. van Es, J.H., et al. 2005. Notch/gamma-secretase inhibition turns proliferative cells in intestinal crypts and adenomas into goblet cells. Nature.
435:959-963

9. Murata, T., Noguchi, P.D., and Puri, R.K. 1996. IL-13 induces phosphorylation and activation of JAK2 Janus kinase in human colon carcinoma cell lines: similarities between IL-4 and IL-13 signaling. J. Immunol. 156:2972-2978.

10. Mathew, A., et al. 2001. Signal transducer and activator of transcription 6 controls chemokine production and $\mathrm{T}$ helper cell type 2 cell trafficking in allergic pulmonary inflammation. J. Exp. Med. 193:1087-1096

11. Kuperman, D.A., et al. 2002. Direct effects of interleukin-13 on epithelial cells cause airway hyperreactivity and mucus overproduction in asthma. Nat. Med. 8:885-889.

12. Finkelman, F.D., et al. 2005. Suppressive effect of 
IL-4 on IL-13-induced genes in mouse lung. J. Immunol. 174:4630-4638.

13. Zhen, G., et al. 2007. IL-13 and epidermal growth factor receptor have critical but distinct roles in epithelial cell mucin production. Am. J. Respir. Cell Mol. Biol. 36:244-253.

14. Wan, H., et al. 2004. Foxa2 regulates alveolarization and goblet cell hyperplasia. Development. 131:953-964.

15. Oettgen, P., et al. 2000. PDEF, a novel prostate epithelium-specific ets transcription factor, interacts with the androgen receptor and activates prostate-specific antigen gene expression. J. Biol. Chem. 275:1216-1225.

16. Park, K.S., et al. 2007. SPDEF regulates goblet cell hyperplasia in the airway epithelium. J. Clin. Invest. 117:978-988.

17. Davies, J.R., Kirkham, S., Svitacheva, N., Thornton, D.J., and Carlstedt, I. 2007. MUC16 is produced in tracheal surface epithelium and submucosal glands and is present in secretions from normal human airway and cultured bronchial epithelial cells. Int J. Biochem. Cell Biol. 39:1943-1954.

18. Komiya, T., Tanigawa, Y., and Hirohashi, S. 1999. Cloning of the gene gob-4, which is expressed in intestinal goblet cells in mice. Biochim. Biophys. Acta. 1444:434-438.

19. Di Valentin, E., et al. 2009. New asthma biomarkers: lessons from murine models of acute and chronic asthma. Am. J. Physiol. Lung Cell Mol. Physiol. 296:L185-L197.

20. Fulkerson, P.C., Fischetti, C.A., Hassman, L.M., Nikolaidis, N.M., and Rothenberg, M.E. 2006. Persistent effects induced by IL-13 in the lung. Am. J. Respir. Cell Mol. Biol. 35:337-346.

21. Kunikata, T., et al. 2005. Suppression of allergic inflammation by the prostaglandin E receptor subtype EP3. Nat. Immunol. 6:524-531.

22. Hattori, R., Shimizu, S., Majima, Y., and Shimizu, T. 2008. EP4 agonist inhibits lipopolysaccharideinduced mucus secretion in airway epithelial cells. Ann. Otol. Rhinol. Laryngol. 117:51-58.

23. Beum, P.V., Basma, H., Bastola, D.R., and Cheng, P.W. 2005. Mucin biosynthesis: upregulation of core 2 beta 1,6 N-acetylglucosaminyltransferase by retinoic acid and Th2 cytokines in a human airway epithelial cell line. Am. J. Physiol. Lung Cell Mol. Physiol. 288:L116-L124.

24. Ross, A.J., Dailey, L.A., Brighton, L.E., and Devlin, R.B. 2007. Transcriptional profiling of mucociliary differentiation in human airway epithelial cells. Am. J. Respir. Cell Mol. Biol. 37:169-185.

25. Huang, J., Olivenstein, R., Taha, R., Hamid, Q., and Ludwig, M. 1999. Enhanced proteoglycan deposition in the airway wall of atopic asthmatics. Am.J. Respir. Crit. Care Med. 160:725-729.

26. Wan, H., et al. 2004. Foxa2 is required for transition to air breathing at birth. Proc. Natl. Acad. Sci. U. S. A. 101:14449-14454.

27. DeFelice, M., et al. 2003. TTF-1 phosphorylation is required for peripheral lung morphogenesis, perinatal survival, and tissue-specific gene expression. J. Biol. Chem. 278:35574-35583.

28. Nakanishi, A., et al. 2001. Role of gob-5 in mucus overproduction and airway hyperresponsiveness in asthma. Proc. Natl. Acad. Sci. U. S. A. 98:5175-5180.

29. Gandhi, R., et al. 1998. Molecular and functional characterization of a calcium-sensitive chloride channel from mouse lung. J. Biol. Chem. 273:32096-32101.

30. Shatos, M.A., et al. 2001. Isolation, characterization, and propagation of rat conjunctival goblet cells in vitro. Invest. Ophthalmol. Vis. Sci. 42:1455-1464.

31. Zeitlmann, L., Grosse, J., Dembowsky, K., and Popp, A. 2006. Methods and agents useful in treating conditions characterized by mucus hyperproduction/hypersecretion. World Intellectual Property Organization patent application PCT/ EP2005/056604, December 8, 2005. Publication WO/2006/061414, June 15, 2006. http://www. wipo.int/pctdb/en/wo.jsp?wo=2006061414\&IA= EP2005056604\&DISPLAY=STATUS.

32. Ramirez, R.D., et al. 2004. Immortalization of human bronchial epithelial cells in the absence of viral oncoproteins. Cancer Res. 64:9027-9034.

33. Chen, H., and Bieberich, C.J. 2005. Structural and functional analysis of domains mediating interaction between NKX-3.1 and PDEF. J. Cell. Biochem. 94:168-177.

34. Yang, Q., Bermingham, N.A., Finegold, M.J., and Zoghbi, H.Y. 2001. Requirement of Math1 for secretory cell lineage commitment in the mouse intestine. Science. 294:2155-2158.

35. Shroyer, N.F., Wallis, D., Venken, K.J., Bellen, H.J., and Zoghbi, H.Y. 2005. Gfi1 functions downstream of Math1 to control intestinal secretory cell subtype allocation and differentiation. Genes Dev. 19:2412-2417

36. Katz, J.P., et al. 2002. The zinc-finger transcription factor Klf4 is required for terminal differentiation of goblet cells in the colon. Development. 129:2619-2628.

37. Guseh, J.S., et al. 2009. Notch signaling promotes airway mucous metaplasia and inhibits alveolar development. Development. 136:1751-1759.

38. Kazanjian, A., et al. 2004. Growth factor independence- 1 is expressed in primary human neuroendocrine lung carcinomas and mediates the differentiation of murine pulmonary neuroendocrine cells. Cancer Res. 64:6874-6882.

39. Gregorieff, A. 2006. Wnt signaling in gut development and homeostasis [dissertation]. Utrecht, The Netherlands: Universiteit Utrecht. http:// igitur-archive.library.uu.nl/dissertations/20060201-200444/full.pdf.

40. Hiemisch, H., Schutz, G., and Kaestner, K.H. 1997. Transcriptional regulation in endoderm development: characterization of an enhancer controlling $\mathrm{Hnf} 3 \mathrm{~g}$ expression by transgenesis and targeted mutagenesis. EMBOJ. 16:3995-4006.

41. Persson, S., et al. 2005. Diversity of the protein disulfide isomerase family: identification of breast tumor induced Hag2 and Hag3 as novel members of the protein family. Mol. Phylogenet. Evol. 36:734-740.

42. Van den Steen, P., Rudd, P.M., Dwek, R.A., and Opdenakker, G. 1998. Concepts and principles of O-linked glycosylation. Crit. Rev. Biochem. Mol. Biol. 33:151-208.

43. Beum, P.V., and Cheng, P.W. 2001. Biosynthesis and function of beta 1,6 branched mucin-type glycans. Adv. Exp. Med. Biol. 491:279-312.
44. Yeh, J.C., Ong, E., and Fukuda, M. 1999. Molecular cloning and expression of a novel beta-1, 6-N-acetylglucosaminyltransferase that forms core 2, core 4, and I branches. J. Biol. Chem. 274:3215-3221.

45. Tan, S., and Cheng, P.W. 2007. Mucin biosynthesis: identification of the cis-regulatory elements of human C2GnT-M gene. Am. J. Respir. Cell Mol. Biol. 36:737-745.

46. Ishibashi, Y., Inouye, Y., Okano, T., and Taniguchi, A. 2005. Regulation of sialyl-Lewis $x$ epitope expression by TNF-alpha and EGF in an airway carcinoma cell line. Glycoconj. J. 22:53-62.

47. Perl, A.K., Zhang, L., and Whitsett, J.A. 2009. Conditional expression of genes in the respiratory epithelium in transgenic mice: cautionary notes and toward building a better mouse trap. Am. J. Respir. Cell Mol. Biol. 40:1-3.

48. Soriano, P. 1999. Generalized lacZ expression with the ROSA26 Cre reporter strain. Nat. Genet. 21:70-71.

49. Betsuyaku, T., and Senior, R.M. 2004. Laser capture microdissection and mRNA characterization of mouse airway epithelium: methodological considerations. Micron. 35:229-234.

50. Xu, Y., Liu, C., Clark, J.C., and Whitsett, J.A. 2006. Functional genomic responses to cystic fibrosis transmembrane conductance regulator (CFTR) and CFTR(delta508) in the lung. J. Biol. Chem. 281:11279-11291.

51. Xu, Y., Ikegami, M., Wang, Y., Matsuzaki, Y., and Whitsett, J.A. 2007. Gene expression and biological processes influenced by deletion of Stat 3 in pulmonary type II epithelial cells. BMC Genomics. 8:455.

52. Wright, G.W., and Simon, R.M. 2003. A random variance model for detection of differential gene expression in small microarray experiments. Bioinformatics. 19:2448-2455.

53. Holzinger, A., et al. 1996. Monoclonal antibody to thyroid transcription factor-1: production, characterization, and usefulness in tumor diagnosis. Hybridoma. 15:49-53

54. Feldman, R.J., Sementchenko, V.I., Gayed, M., Fraig, M.M., and Watson, D.K. 2003. Pdef expression in human breast cancer is correlated with invasive potential and altered gene expression. Cancer Res. 63:4626-4631.

55. Schambach, A., et al. 2006. Equal potency of gammaretroviral and lentiviral SIN vectors for expression of O6-methylguanine-DNA methyltransferase in hematopoietic cells. Mol. Ther. 13:391-400.

56. You, Y., Richer, E.J., Huang, T., and Brody, S.L. 2002. Growth and differentiation of mouse tracheal epithelial cells: selection of a proliferative population. Am. J. Physiol. Lung Cell Mol. Physiol. 283:L1315-1321.

57. Vaughan, M.B., Ramirez, R.D., Wright, W.E., Minna, J.D., and Shay, J.W. 2006. A three-dimensional model of differentiation of immortalized human bronchial epithelial cells. Differentiation. 74:141-148.

58. Matsui, H., Randell, S.H., Peretti, S.W., Davis, C.W., and Boucher, R.C. 1998. Coordinated clearance of periciliary liquid and mucus from airway surfaces. J. Clin. Invest. 102:1125-1131.

59. Hsu, J.C. 1981. Simultaneous confidence intervals for all distances from the "best." Annals of Statistics. 9:1026-1034 